\title{
61. On the Evolution Equations with Finite Propagation Speed
}

\author{
By Sigeru MizohatA
}

(Comm. by Kinjirô Kunugr, M. J. A., March 12, 1970)

1. Introduction. Let

$$
\left(\frac{\partial}{\partial t}\right)^{m} u(x, t)=\sum_{j<m} a_{\nu j}(x, t)\left(\frac{\partial}{\partial x}\right)^{\nu}\left(\frac{\partial}{\partial t}\right)^{j} u(x, t)
$$

be an evolution equation defined on $(x, t) \in \boldsymbol{R}^{l} \times[0, T] \equiv \Omega$. We suppose all the coefficients are infinitely differentiable, and that for any time $t_{0} \in[0, T)$ and any initial data

$$
\left(\frac{\partial}{\partial t}\right)^{j} u\left(x, t_{0}\right)=\varphi_{j}(x) \in \mathscr{D}(j=0,1, \cdots, m-1),
$$

there exists a unique solution $u(x, t)$ for $t \in\left[t_{0}, T\right]$ in some functional space, say in $\mathcal{B}$ or in $\mathscr{D}_{L} p(1<p<+\infty)$. $^{1)}$

We say that (1.1) has a finite propagation speed if for any compact $K$ in $\boldsymbol{R}^{l}$, there exists a finite $\lambda(K)$ (propagation speed) such that for any initial data $\Psi(x) \equiv\left(\varphi_{0}(x), \cdots, \varphi_{m-1}(x)\right) \in \mathscr{D}$, with initial time $t_{0}$, whose support is contained in $K$, the support of the solution $u(x, t)$ is contained in

$$
\bigcup_{\xi \in \sup [\Psi]}\left(\xi, t_{0}\right)+C_{\lambda(K)}^{+},
$$

where $C_{\lambda(K)}^{+}$is the cone defined by $\{(x, t) ;|x| \leq \lambda(K) t, t \geq 0\}$.

We say that (1.1) is a kowalevskian in $\Omega$, if the coefficients $a_{\nu j}(x, t)$ appearing in the second member are identically zero if $|\nu|+j>m$. Our result is the

Theorem. In order that (1.1) have a finite propagation speed, it is necessary that (1.1) be kowalevskian in $\Omega$.

This theorem was proved by Gårding [1] in the case where all the coefficients are constant. Now we can prove this theorem by the same method as in [2]. The detailed proof will be given in a forthcoming paper. In this Note, to make clear our reasoning, we argue on a simple equation.

2. Localizations of equation. Let

$$
\frac{\partial}{\partial t} u(x, t)=\sum_{|\nu| \leq p} a_{\nu}(x, t)\left(\frac{\partial}{\partial x}\right)^{\nu} u(x, t) \equiv a_{p}\left(x, t ; \frac{\partial}{\partial x}\right) u(x, t)
$$

be an evolution equation, not kowalevskian, in $\Omega$. Without loss of generality, we may assume that at the origin the second member of (2.1) is effectively of order $p(>1)$. We can find then a complex num-

1) With regards to these notations, see [2]. As the proof given later shows, this conditions can be replaced by weaker conditions. 
ber $\zeta_{0}=\xi_{0}+i \eta_{0}\left(\xi_{0}, \eta_{0} \neq 0\right)$, such that

$$
\operatorname{Re} \sum_{|\nu|=p} a_{\nu}(0,0) \zeta_{0}^{\nu}=2 \delta>0 \text {. }
$$

Now take a function $\beta(x) \in \mathscr{D}$ of small support taking the value 1 in a neighborhood of $x=0$. Apply $\beta(x)$ to (2.1), then

$$
\frac{\partial}{\partial t}(\beta u)=a_{p}\left(x, t ; \frac{\partial}{\partial x}\right)(\beta u)+\sum_{\mu} a_{p, \mu}\left(x, t ; \frac{\partial}{\partial x}\right)\left(\beta^{(\mu)} u\right),
$$

where the coefficients may be supposed, by changing these outside the support of $\beta(x)$, to be near the values at the origin (localization in the $x$-space) if we restrict the variable $t$ to a small neighborhood of zero, say $t \leq \varepsilon$. Here the order of $a_{p, \mu}$ is equal to $(p-|\mu|)$.

Now by the hypothesis of the well-posedness of (2.1), there exists a constant $C$ and $h$ independent of $\left(x_{0}, t_{0}\right)$ such that it hold for any initial data $u(x, 0) \in \mathscr{D}$,

$$
\begin{aligned}
\left|u\left(x_{0}, t_{0}\right)\right| & \leq C \sum_{|\alpha| \leq h} \sup _{x \in R^{l}}\left|D^{\alpha} u(x, 0)\right|, \text { or } \\
& \leq C \sum_{|\alpha| \leq h}\left\|D^{\alpha} u(x, 0)\right\|_{L^{p}\left(\boldsymbol{R}^{l}\right)},
\end{aligned}
$$

for any $x_{0} \in \operatorname{supp}[\beta]$ and $t_{0} \in[0, T]$. So, let us denote by $T_{y}\left(x_{0}, t_{0}\right)$ the distribution (in $y$ ) defined by

$$
u\left(x_{0}, t_{0}\right)=\left\langle T_{y}\left(x_{0}, t_{0}\right), u(y, 0)\right\rangle \text {. }
$$

Let us suppose that (2.1) has a finite propagation speed. This implies that there exists a positive constant $\lambda$ such that for $x_{0} \in \operatorname{supp}[\beta]$, and $t_{0} \in[0, \varepsilon]$, ( $\varepsilon$ small),

$$
\operatorname{supp}\left[T_{y}\left(x_{0}, t_{0}\right)\right] \subset B_{\lambda t_{0}}\left(x_{0}\right) \equiv\left\{y ;\left|y-x_{0}\right| \leq \lambda t_{0}\right\} \text {. }
$$

Now in any case of (2.4), it is shown that we can sharpen the inequality (2.4) in the following way:

$$
\left|\left\langle T_{y}\left(x_{0}, t_{0}\right), u(y, 0)\right\rangle\right| \leq C^{\prime} \sum_{|\alpha| \leq h} \sup _{\left|y-x_{0}\right| \leq \lambda t_{0}}\left|D^{\alpha} u(y, 0)\right|,
$$

where $C^{\prime}$ depends on $C, h$ and $l$, but does not depend on $\left(x_{0}, t_{0}\right)$.

Let $\hat{u}_{0}(\eta)$ be a continuous function $\neq 0$ whose support is contained in a unit sphere with center at the origin, and let $u_{0}(x)$ be the inverse Fourier image. We define a sequence of solutions $u_{n}(x, t)$ of (2.1) by the initial data,

$$
u_{n}(x, 0)=\gamma(x) e^{n x \cdot \xi_{0}} u_{0}(x) \equiv \gamma(x) e^{n x \cdot \xi_{0}} e^{i n x \cdot \eta_{0}} u_{0}(x) \in \mathscr{D},
$$

where $\gamma(x)$ is a function of $\mathscr{D}$ which takes the value 1 on the set $|x|$ $\leq L$ (sufficiently large).

Next apply $e^{-n x \cdot \xi_{0}}$ to (2.3) after replacing $u$ by $u_{n}$, it becomes

$$
\begin{aligned}
\frac{\partial}{\partial t}\left(\beta e^{-n x \cdot \xi_{0}} u_{n}\right)= & a_{p}\left(x, t ; \frac{\partial}{\partial x}+n \xi_{0}\right)\left(\beta e^{-n x \cdot \xi_{0}} u_{n}\right) \\
& +\sum a_{p, \mu}\left(x, t ; \frac{\partial}{\partial x}+n \xi_{0}\right)\left(\beta^{(\mu)} e^{-n x \cdot \xi_{0}} u_{n}\right) .
\end{aligned}
$$

Now let us estimate the function

$$
v_{n}(x, t)=e^{-n x \cdot \xi_{0}} u_{n}(x, t) .
$$

By (2.7), 
So we have

$$
\begin{aligned}
\left|e^{-n x \cdot \xi_{0}} u_{n}(x, t)\right| & =\left|\left\langle T_{y}(x, t), e^{-n x \cdot \xi_{0}} \gamma(y) e^{i n y \cdot \xi_{0}} u_{0}(y)\right\rangle\right| \\
& =\left|\left\langle T_{y}(x, t), e^{n(y-x) \cdot \xi_{0}} e^{i n y \cdot \eta_{0}} u_{0}(y)\right\rangle\right| \\
& \leq C^{\prime} \sum_{|\alpha| \leq h} \sup _{|y-x| \leq \lambda t}\left|D^{\alpha}\left\{e^{n(y-x) \cdot \xi_{0}} e^{i n y \cdot \eta_{0}} u_{0}(y)\right\}\right| .
\end{aligned}
$$

$$
\left|v_{n}(x, t)\right| \leq C^{\prime \prime} n^{h} \exp \left(n \lambda\left|\xi_{0}\right| t\right), \quad \text { for } \quad x \in \operatorname{supp}[\beta]
$$

and $t \in[0, \varepsilon]$,

where $C^{\prime \prime}$ is a constant independent of $(x, t)$ and $n$. Remarking this, let $\alpha(\eta)$ be a function of $\mathscr{D}$ having its support in a small neighborhood of $\eta_{0}$, and taking the value 1 in a neighborhood of $\eta_{0}$. Finally, putting

$$
\alpha_{n}(\eta)=\alpha(\eta / n)
$$

we define the convolution operator $\alpha_{n}(D)$. Applying this to (2.8), we get a new equation localized in both $x$ and $\eta$ spaces :

$$
\begin{aligned}
\frac{\partial}{\partial t}\left(\alpha_{n}(D) \beta v_{n}\right)= & a_{p}\left(x, t ; \frac{\partial}{\partial x}+n \xi_{0}\right)\left(\alpha_{n}(D) \beta v_{n}\right) \\
& +\sum a_{p, \mu}\left(x, t ; \frac{\partial}{\partial x}+n \xi_{0}\right)\left(\alpha_{n}(D) \beta^{(\mu)} v_{n}\right) \\
& +\left[\alpha_{n}(D), a_{p}\right]\left(\beta v_{n}\right)+\sum\left[\alpha_{n}(D), a_{p, \mu}\right]\left(\beta^{(\mu)} v_{n}\right) .
\end{aligned}
$$

3. Energy inequality. Let us consider the following equation:

$$
\frac{\partial}{\partial t}\left(\alpha_{n}(D) w(x, t)\right)=a_{p}\left(x, t ; \frac{\partial}{\partial x}+n \xi_{0}\right)\left(\alpha_{n}(D) w\right)+f(x, t) .
$$

Taking account of (2.2), it is shown that the following inequality holds for $t \in[0, \varepsilon]$ :

$$
\frac{d}{d t}\left\|\alpha_{n}(D) w(x, t)\right\| \geq \delta n^{p}\left\|\alpha_{n}(D) w(x, t)\right\|-\|f(x, t)\|,
$$

where $\|\cdot\|$ denotes the $L^{2}$-norm in $\boldsymbol{R}^{l}$. In fact, on the support of $\alpha_{n}(\eta)$, the symbol of $a_{p}\left(x, t ; \frac{\partial}{\partial x}+n \xi_{0}\right)$ behaves like $a_{p}\left(x, t ; n \zeta_{0}\right)$. Now, in view of (2.11), we have

$$
\left|\alpha_{n}^{(\kappa)}(\eta)\right| \leq \text { constant. } n^{-|\kappa|}
$$

So, if we develop the commutator $\left[\alpha_{n}(D), a_{p}\right]$, it holds:

$$
\left[\alpha_{n}, a_{p}\right]=\sum_{|\kappa|=1}^{m} i^{|\kappa|} \partial_{x}^{s} a_{p}\left(x, t ; \frac{\partial}{\partial x}+n \xi_{0}\right) \alpha_{n}^{(\kappa)}(D)+R_{m, p},
$$

where

$$
\left\|R_{m, p}(u)\right\| \leq \text { constant. } n^{l+p-m-1}\|u\| \text {, }
$$

where, let us recall, $l$ is the dimension of the space and $p$ is the order of $a_{p}$. The same kind of inequalities holds for $\left[\alpha_{n}, a_{p, \mu}\right]$. So, if we take

$$
m=h+l \text {, }
$$

we shall have, in view of (2.10), (2.12) and (3.2):

$$
\begin{aligned}
& \frac{d}{d t}\left\|\alpha_{n} \beta v_{n}\right\| \geq \delta n^{p}\left\|\alpha_{n} \beta v_{n}\right\|-c n^{p} \sum_{1 \leq|\kappa| \leq m}\left\|\alpha_{n}^{(k)} \beta v_{n}\right\| \\
& \quad-c n^{p-1} \sum_{1 \leq|\kappa| \leq m-1,|\mu|=1}\left\|\alpha_{n}^{(k)} \beta^{(\mu)} v_{n}\right\|-\cdots-c n^{p-i} \sum_{1 \leq|\kappa| \leq m-i,|\mu|=i}\left\|\alpha_{n}^{(k)} \beta^{(\mu)} v_{n}\right\|
\end{aligned}
$$




$$
\cdots-c \sum_{|\mu|=p}\left\|\alpha_{n} \beta^{(\mu)} v_{n}\right\|-c n^{p-1} \exp \left(n\left|\xi_{0}\right| \lambda t\right) .
$$

Namely

$$
\begin{aligned}
\frac{d}{d t}\left\|\alpha_{n} \beta v_{n}\right\| \geq & \delta n^{p}\left\|\alpha_{n} \beta v_{n}\right\|-c n^{p} \sum_{1 \leq|x|+|\mu| \leq m}\left\|\alpha_{n}^{(k)} n^{-|\mu|} \beta^{(\mu)} v_{n}\right\| \\
& -c n^{p-1} \exp \left(n\left|\xi_{0}\right| \lambda t\right)
\end{aligned}
$$

Define

$$
S_{n}(t)=\sum_{|\kappa|+|\mu| \leq m} C_{0}^{|\kappa|+|\mu|}\left\|\alpha_{n}^{(\kappa)} n^{-|\mu|} \beta^{(\mu)} v_{n}\right\|
$$

This means that we consider all the functions $\alpha_{n}^{(\kappa)} \beta^{(\mu)} v_{n}$ instead of $\alpha_{n} \beta v_{n}$ in (2.12). Then we shall have the same kinds of inequalities as (3.4). So, if we choose $C_{0}$ large enough, summing up all the inequalities thus obtained, we shall have

$$
\mathrm{S}_{n}^{\prime}(t) \geq \frac{\delta}{2} n^{p} S_{n}(t)-c^{\prime} n^{p-1} \exp \left(n\left|\xi_{0}\right| \lambda t\right) .
$$

\section{Hence}

$$
\begin{aligned}
S_{n}(t) \geq S_{n}(0) \exp ( & \left.\frac{\delta}{2} n^{p} t\right) \\
& -c^{\prime} n^{p-1} \exp \left(\frac{\delta}{2} n^{p} t\right) \int_{0}^{t} \exp \left(-\frac{\delta}{2} n^{p} \tau\right) \exp \left(n\left|\xi_{0}\right| \lambda \tau\right) d \tau .
\end{aligned}
$$

Taking account of $\left\|\alpha_{n}(D) \beta(x) v_{n}(x, 0)\right\|=\left\|\alpha_{n}(D) \beta(x) e^{i n x \cdot \eta_{0}} u_{0}(x)\right\|$, and in view of [2], we see that $\left\|\alpha_{n} \beta v_{n}(x, 0)\right\| \geq \delta_{0}(>0)$ for $n$ large. A fortiori, it holds $S_{n}(0) \geq \delta_{0}$ for $n$ large. Thus,

$$
S_{n}(t) \geq \frac{\delta_{0}}{2} \exp \left(\frac{\delta}{2} n^{p} t\right) \text { for } t \in[0, \varepsilon], \quad n \text { large. }
$$

In fact, for $n$ large, since $p>1$, we have $n\left|\xi_{0}\right| \lambda<\frac{\delta}{4} n^{p}$. Then

$$
\begin{aligned}
\int_{0}^{t} \exp \left(-\frac{\delta}{2} n^{p} \tau\right) \exp \left(n\left|\xi_{0}\right| \lambda \tau\right) d \tau & \leq \int_{0}^{t} \exp \left(-\frac{\delta}{4} n^{p} \tau\right) d \tau \\
& \leq \frac{1}{n^{p}} \int_{0}^{\infty} \exp \left(-\frac{\delta}{4} \tau\right) d \tau .
\end{aligned}
$$

On the other hand, (2.10) shows that $S_{n}(t) \leq$ const. $n^{h} \exp \left(n\left|\xi_{0}\right| \lambda t\right)$. This inequality is not compatible with (3.5) unless $t=0$. Thus we proved the Theorem in the Introduction by contradiction.

\section{References}

[1] L. Gårding: Linear hyperbolic partial differential equations with constant coefficients. Acta Math., 85, 1-62 (1951).

[2] S. Mizohata: Some remarks on the Cauchy problem. J. of Math. Kyoto Univ., 1, 109-127 (1961). 